\title{
Software for Time Series Analysis
}

\section{G. Mélard, Free University of Brussels, Belgium}

Summary : This paper is devoted to the presentation of (a) a general approach in univariate time series analysis, and (b) the corresponding computer software called ANSECH. The methodology is similar to that of Box and Jenkins(1970) but the class of models is wider. The main algorithms for estimation and forecasting are briefly described.

Keywords : time series, Box and Jenkins method, statistical algorithms.

1. Introduction

Given a time series $w=\left\{w_{t} ; t=1, \ldots, n\right\}$, a parametric model belonging to the class of stationary and invertible autoregressive-moving average (ARMA) models is searched for. The model is defined by

$w_{t}=\phi_{1} w_{t-1}+\ldots+\phi_{p} w_{t-p}+a_{t}-\theta_{1} a_{t-1}-\ldots-\theta_{q} a_{t-q}$,

$t \in \mathbb{Z}, a=\left\{a_{t} ; t=1, \ldots, n\right\}$ being a white-noise process. Since the book of Box and Jenkins(1970), the tools of the time-series analyst have been improved, see the review by Newbold(1981). Many authors, including Box and Jenkins themselves, have admitted that the lack of stationarity of many time series implies that extensions of ARMA models should be used. Most computer programs on the market have few of these extensions. In this paper, we will describe a comprehensive class of models supported by a software called ANSECH (ANalyse des SEries CHronologiques), various estimation procedures and some aspects of the computer program.

Hipel and McLeod(1980) and Khabie-Zeitoune(1982) have had similar concerns.

\section{The class of models}

We will use the following notations :

(a) $y_{t}^{I}, y_{t}^{D}, y_{t}^{W}, y_{t}^{M}$ and $y_{t}^{S}$ are arbitrary functions of $t$;

(b) $f_{t}$ and $g_{t}$ are positive functions of $t$;

(c) $\mu_{t}$ and $\mu_{t}^{\prime}$ are polynomial functions of $t$;

(d) $m_{t}$ and $m_{t}^{\prime}$ are periodic functions of $t$;

(e) $\phi_{i t}$ and $\theta_{j t}$ are either constants $\left(\phi_{i}, \theta_{j}\right)$, or functions of $t$;

(f) $C($.$) defines a transformation which is invariant with repect to t$;

(g) $\Phi_{i}$ and $\xi_{j}$ are constants.

We denote by ${ }^{j} d, D, p, q, P, Q, s$, non-negative integers, $s \neq 0$. Let $\nabla=1-B$ and $\nabla_{S}=1-B^{S}$, where $B$ is the backshift operator $\left(B \cdot_{t}=t_{t-1}\right)$. Finally, $z=\left\{z_{t}\right.$; $1-d-D s \leqslant t \leqslant n\}$ is a time series and $a=\left\{a_{t} ; 1 \leqslant t \leqslant n\right\}$ is seen as a realization of a white-noise process with mean 0 and variance $\sigma^{2}$.

The following equations, called respectively the variable sub-model, the exten- 
ded ARMA sub-model and the innovation sub-model, yield $a_{t}$ from the $z_{t}$ :

$w_{t}=\nabla^{d} \nabla_{s}^{D}\left[C\left(z_{t}-y_{t}^{I}\right) / f_{t}\right]-\left(y_{t}^{D}+\mu_{t}+m_{t}\right)$

$b_{t}=\frac{w_{t}-\Sigma p_{i=1}^{p} \phi_{i t} w_{t-i}+\Sigma g_{j=1}^{g} \theta_{j t} b_{t-j}+y_{t}^{W}}{1+\Sigma{ }_{i=1}^{p} \tilde{\phi}_{j} w_{t-j}-\Sigma \sum_{j=1}^{Q} \tilde{\theta}_{j} b_{t-j}}$

$a_{t}=\exp \left(-y_{t}^{S}\right)\left[b_{t} / g_{t}-\left(y_{t}^{M}+\mu_{t}^{1}+m_{t}^{\prime}\right)\right]$.

The subscript $t$ will be deleted when the context is clear. The parameters of the model are $\sigma^{2}$ and those included in $(a-g)$. The analytical form of the functions (a-f) must be specified. The following remarks are intended to justify (2-4) :

$1^{\circ}$ Functions (a) can describe the effects of interventions. In particular, $y$ has been used by Box and Tiao(1975) and $y^{S}$ is concerned with the innovation variance. Instead of relying on transfer function models, a parametrization via piece-wise linear functions is used (Mélard,1981). This is much more efficient in all respects, e.g. when there are several outliers.

$2^{\circ}$ Several options exist for (b) in order to model a trend on the scale parameter of the time series (Mélard,1977).

$3^{\circ}$ Functions $(c-d)$ can be used when deterministic trend or seasonal are better than the corresponding stochastic components provided by differencing. The seasonal component can be parcimoniously parametrized in terms of seasonal coefficients, Fourier coefficients (Abraham and Box, 1978) or a mixture of both.

$4^{\circ}$ The introduction of $\mu^{\prime}$ and $\mathrm{m}^{\prime}$ in (c-d) has been examined by Mélard(1982a).

$5^{\circ}$ In the extended ARMA sub-model, the coefficients $\phi_{i t}$ and $\theta_{j t}$ can vary linearly or exponentially with $t$ (Mélard and Kiehm, 1981). It is also possible to handle the natural parameters of various short-term forecasting methods (Mélard and Rouland, 1979).

$6^{\circ}$ The transformation (f) is based on the power family as in Box and Cox(1964) and Ansley et al (1977) :

$C\left(x_{t}\right)=\lambda^{-1}\left(x_{t}^{\lambda}-1\right)$ for $\lambda \neq 0, c\left(x_{t}\right)=\log x_{t}$ for $\lambda=0$.

$7^{\circ}$ The non-linear model including $(g)$ has been used by Lentz and Mélard(1981). The most useful elements among these extensions appear to be : (a) $y^{I}, y^{D}$ and $y^{S}$; (b) $g$ and (f).

\section{Estimation of the parameters of the extended model}

Let $\alpha$ be the vector of all the parameters except $\sigma^{2}$, and assume that the $a_{t}$ are n.i.d. $\left(0, \sigma^{2}\right)$. The likelihood function, conditional on $a_{t}$ and $z_{t}, t \leqslant 0$, is given by

$L\left(\alpha, \sigma^{2} ; z\right)=(2 \pi)^{-n / 2} J(\alpha) \sigma^{-n} \exp \left\{-\frac{1}{2 \sigma^{2}} \sum_{t=1}^{n} a_{t}^{2}(\alpha)\right\}$,

where $a_{t}(\alpha)$ is derived from $(2-4)$ and the Jacobian $J(\alpha)=\left|\left(\partial z_{t} / \partial a_{s}\right)\right|$ is 
$J(\alpha)=\prod_{t=1}^{n}\left\{f_{t} g_{t} \exp \left(y_{t}^{S}\right)\left|1+\Sigma_{i} \tilde{\phi}_{i} w_{t-i}-\Sigma_{j} \tilde{\theta}_{j} b_{t-j}\right|\left(z_{t}-y_{t}^{I}\right)^{\lambda-1}\right\}$.

By letting $\sigma_{*}=\sigma(J(\alpha))^{1 / n}$, the concentrated likelihood is

$(2 \pi)^{-n / 2} \sigma_{*}^{-n} \exp \left\{-S(\alpha) /\left(2 \sigma_{*}^{2}\right)\right\}$, where $S(\alpha)=\Sigma\left\{a(\alpha) /(J(\alpha))^{1 / n}\right\}^{2}$.

Maximizing (5) is equivalent to finding $\hat{\alpha}$ such that $S(\hat{\alpha})$ is minimum. Then an estimate $\hat{\sigma}^{2}$ of $\sigma^{2}$ is deduced from $\hat{\sigma}_{*}^{2}=S(\hat{\alpha}) / \mathrm{n}$. In practice, the constraints $\pi f_{t}=$ II $g_{t}=\pi \exp \left(y_{t}^{S}\right)=1$ are imposed and the Box-Cox transformation is normalized.

Brubacher (1977) has noted that if $\lambda$ is included in the model, the information matrix may not be estimated by

$\hat{\sigma}^{2} \Sigma \frac{\partial a_{t}}{\partial \alpha^{\prime}} \frac{\partial a_{t}}{\partial \alpha}$ but by $\hat{\sigma}^{2} \Sigma\left\{\frac{\partial a_{t}}{\partial \alpha^{\prime}} \frac{\partial a_{t}}{\partial \alpha}+a_{t} \frac{\partial^{2} a_{t}}{\partial \alpha^{\prime} \partial \alpha}\right\}$.

The same is true when $f, g$ or $y^{S}$ contains parameters.

The algorithm for computing a by the conditional method is divided in 14 steps. The steps 1 to $(r-1)$ are executed only once, where the $r$-th step is the first in which parameters are involved. The optimization routine calls steps $r$ to 14 with the current parameters values, as many times as necessary. Only three auxiliary arrays $X 1, X 2$ and $X 3$, with length not greater than $(n+d+D s)$, are needed. Let $X 4$ contain a. The algorithm is summarized in Table 1.

Table 1.- Algorithm for computing a by the conditional method

Exit : $Y($ es) if parameters first encountered at the next step, $N(0)$ otherwise Entry : $Y$ if parameters first encountered at the current step, $N$ otherwise Parameters : indicates where they are included $X 1, X 2, X 3, X 4$ : operations performed (by default : on the same array) List of operators : (op) $\epsilon\left\{=,+,-,,, /, \nabla^{d}, \nabla_{S}, A R M A\right\}$ An operation in column $X_{i}$ should be understood as an assignment $X_{i}=: X_{i}(o p)$ operand, except for "=" which is : $X_{i=: \text { operand. }}$

\begin{tabular}{|c|c|c|c|c|c|c|c|}
\hline Step & Exit & Entry & Parameters & $X 1$ & $x 2$ & $x 3$ & $\times 4$ \\
\hline 0 & $y$ & $Y$ & & $=z_{I}$ & & & \\
\hline 1 & $\begin{array}{l}y \\
y\end{array}$ & y & $y^{I}$ & $=-y^{-1}$ & & & \\
\hline 3 & $\mathrm{~N}$ & y & & $=c()$. & & $=f$ & \\
\hline 4 & $\mathrm{~N}$ & $\mathrm{~N}$ & & $\nabla d^{d}$ & & & \\
\hline 5 & $Y$ & $\mathrm{~N}$ & & $\nabla \rho_{n}$ & & & \\
\hline 6 & $Y$ & Y & $y^{D}, y^{M}$ & $-y^{D}$ & $=X 1$ & & \\
\hline 7 & Y & Y & $\mu, \mu^{\prime}$ & $-\mu$ & & & \\
\hline 8 & $y$ & Y & $m, m^{\prime \prime}$ & $-m$ & & & \\
\hline $\begin{array}{r}9 \\
10\end{array}$ & $\begin{array}{l}Y \\
N\end{array}$ & $\begin{array}{l}y \\
y\end{array}$ & $\phi, \theta, \phi, \theta$ & & & $=a$ & \\
\hline 11 & Y & $\mathrm{N}$ & & & & & \\
\hline 12 & $\mathrm{~N}$ & Y & $y^{W}, y^{S}$ & & & & \\
\hline 13 & $\mathrm{~N}$ & $\mathrm{~N}$ & & & $\begin{array}{c}=-y^{n} \\
\operatorname{ARMA}(X 1)\end{array}$ & & \\
\hline 14 & Y & $\mathrm{N}$ & & & $\int^{2}$ & $\checkmark$ & $\begin{array}{l}=\times 2 / X 33-\left(y^{M}+\mu^{\prime}+m^{\prime}\right) \\
/ \exp \left(y^{\prime}\right)\end{array}$ \\
\hline
\end{tabular}


Forecasting can be performed in two stages :

$1^{\circ}$ recovery of intermediary arrays, see Table 2 ;

$2^{\circ}$ computation of the forecasts, see Table 3 . Four other arrays are necessary, denoted by $\times 5, X 6, X 7$ and $\times 8$.

Table 2.- Algorithm for forecasting : stage 1.

The macro-instruction $[1, \mathrm{~m}]\left(\mathrm{X}_{1}, \mathrm{X} 2, \mathrm{X} 3, \mathrm{X} 4\right)$ means that steps 1 to $\mathrm{m}$ of the algorithm of Table 1 are executed.

\begin{tabular}{l|l} 
Instruction & Result \\
\hline$[1,11](X 1, \times 2, X 3, X 4)$ & $X 1=w$ \\
{$[1,6](X 4, X 2, X 3, X 4)$} & $X 2=w+y^{D}+\mu+m$ and $X 3=f$ \\
$X 2=: X 2-X 1$ & $X 2=y^{D}+\mu+m$ \\
{$[1,1](X 1, X 4, X 4, X 4)$} & $X 1=z-y^{I}$ \\
$X 4=: z-X 1$ & $X 4=y^{I}$ \\
{$[1,3](X 1, X 3, X 3, X 3)$} & $X 1=C\left(z-y^{I}\right) / f$
\end{tabular}

Table 3.- Algorithm for forecasting : stage 2.

The distinction between the observation and the forecast intervals is made by the concatenation operator ";"

\begin{tabular}{l|l} 
Instruction & Result \\
\hline$[1,14](X 5, X 6, X 7, X 8)$ & $X 5=w$ \\
$X 8=: w+X 2$ & $X 8=w+y^{D}+\mu+m$ \\
$X 8=: X 1 ; X 8$ & $X 8=C\left(z-y^{I}\right) / f ; w+y^{D}+\mu+m$ \\
$X 8=: X 8 ;\left(\nabla^{d} \nabla_{S}^{D}\right)-1 \times 8$ & $X 8=C\left(z-y^{I}\right) / f$ \\
$X 8=: C^{-1}(X 8 . X 3)+X 4$ & $X 8=z$
\end{tabular}

Three other estimation methods can be used in the program :

$1^{\circ}$ The unconditional method, with the backforecasting procedure (Box and Jenkins, 1970), iterated on request. The algorithm is more complex than the algorithm for the conditional method because of the time-dependency of the coefficients $\phi$ and $\theta$ (Kiehm et al,1980) and presence of $\mu^{\prime}$ and $m^{\prime}$ (Mélard,1982a).

$2^{\circ}$ The exact likelihood method for ARMA models with constant coefficients, without $y^{S}, g, \mu^{\prime}, m^{\prime}, \tilde{\phi}, \tilde{\theta}$. The algorithm (Mélard,1982c) is faster than the approximate unconditional method.

$3^{\circ}$ The exact likelihood method for ARMA models with time-dependent models, without $\mu^{\prime}, m^{\prime \prime}, \tilde{\phi}, \tilde{\theta}$ (Mélard,1982b).

We will not give here the corresponding modifications to Table 1 . In all cases, a sum of squares $S(\alpha)$ is minimized with respect to $\alpha$ by the Marquardt algorithm. 


\section{General description of the software}

In the sequel of this paper we will mention a number of features provided by the computer program ANSECH (Mêlard, 1975), written in Extended Fortran C.D.C. for a Cyber 170/750 system. Each problem is decomposed into sections, as shown in Table 4. The problem is described by a command sentence using separated commands of the form "identifier" or "identifier=option". Each identifier is associated with two default option values, one to be supplied when the identifier is absent from the command sentence, the other, when the first form of the command is used. Examples of identifiers are shown in Table 4.

Table 4.- Decomposition of a problem into sections, with some examples of commands

\begin{tabular}{|c|c|c|}
\hline Section & |Purpose & Examples of commands \\
\hline A & input validation & ESTIM must be present if estimation is requested \\
\hline B & access to data & $K E Y=$ name of data set (in case of random access) \\
\hline C & model definition & DIFF $=$ degree of regular difference (value of $d)$ \\
\hline & & BOXCOX for normalized logarithmic transformation \\
\hline D & parameter meaning & HOLT $=1$ or 2 Holt or Winters model (instead of ARMA) \\
\hline E & estimation & $\begin{array}{l}\text { ITERATIONS = maximum number of iterations (Marquardt) } \\
\text { LIKELIHOOD = estimation method }\end{array}$ \\
\hline $\mathrm{F}$ & plots, diagnostics & $\begin{array}{l}\text { CHECKING = a string of code letters defining the } \\
\text { requests ( } A \text { for autocorrelation, } L \text { for } L \text { jung-Box,...) }\end{array}$ \\
\hline G & forecasting & $\begin{array}{l}\text { LEADTIME = number of steps ahead for which forecasts } \\
\text { are asked for }\end{array}$ \\
\hline
\end{tabular}

Further lines of directives are required for

- preliminary transformations,

- selection of parameters : type, order, initial value,

- description of interventions (Mélard, 1981).

There are fourteen types of parameters, plus the various parameters in the intervention directives. For example, the letters $M$ and $N$ identify respectively the regular and seasonal moving average polynomials. The following two lines can be used to fit the airline passengers data (Box and Jenkins, 1970, Ch 9) by the model

$$
\nabla \nabla_{12} \log z=a_{t}-\theta_{1} a_{t-1}-\theta_{1}{ }_{t-12}+\theta_{1} \theta_{1} a_{t-13}
$$

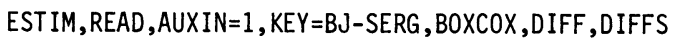

\section{$\begin{array}{lllllll}M & 1 & 0.5 & N & 1 & 0.6\end{array}$}

("READ,AUXIN=1" specifies reading from input file $\# 1$, DIFFS corresponds to $\nabla_{S}$ ).

The command language is easy to learn, as observed with about a hundred students and researchers, many of them without any experience in data processing. However, a program has been written recently that questions the user who wishes to enter new 
data or solve simple problems. Command sentences are composed, shown to the user, who can supplement them.

It is not possible to detail other features of ANSECH such as : data management, dating facility, extended residual tests, calculation of the likelihood on grids of points, transformations, Monte Carlo processor (which can yield a ready-to-use SPSS input file).

\section{References}

Abraham, B. and Box, G.E.P. (1978), Deterministic and forecast-adaptative timedependent models, J.Roy.Statist.Soc.Ser.C Appl.Statist., 27,120-130.

Anderson, O.D. and Perryman, M.R. (eds)(1981), Time series analysis, North-Holland, Amsterdam.

Ansley, C.F., Spivey, W.A. and Wrobleski, W.J.(1977), A class of transformations for Box-Jenkins seasonal models, J.Roy. Statist.Soc.Ser. C Appl.Statist., 26, 173-178.

Box, G.E.P. and Cox, D.R.(1964), An analysis of transformations, J.Roy. Statist.Soc. Ser. B,26,211-243.

Box, G.E.P. and Jenkins, G.M.(1970), Time series analysis, forecasting and control, Holden-Day, San Francisco(revised edition : 1976).

Box, G.E.P. and Tiao, G.C.(1975), Intervention analysis with applications to economic and environmental problems, J.Am.Statist. Assoc.,70,70-79.

Brubacher, S.R.(1977), Applications and extensions of the Box-Jenkins model, Communication at the Public Utilities Forecasting Conference, Browness-on-Windermere.

Hipe1, K.W. and McLeod, A.I.(1980), Perspectives in stochastic hydrology, in 0.D. Anderson (ed), Time series, North-Holland, Amsterdam,73-102.

Khabie-Zeitoune, E.(1982), Identification, estimation and prediction of 1 inear and nonlinear multivariate models in time series, Proceedings of the 4th International Time Series Meeting, Valencia, June 1981, 0.D. Anderson(ed), North-Holland, Amsterdam, to appear.

Kiehm, J.-L., Lefèvre, C. and Mélard, G.(1980), Forme rétrospective d'un modèle FARIMAG, Cahiers du C.E.R.0., 22,375-383.

Lentz, J.-R. and Mélard, G.(1981), Statistical analysis of a non-linear time series model, in 0.D. Anderson and M.R. Perryman(eds)(1981), 287-293.

Mélard, G.(1975), ANSECH-Programmes pour l'analyse des séries chronologiques, Presses Universitaires de Bruxelles, Bruxelles(4th edition : 1981).

Mélard, G. (1977), Sur une classe de modèles ARIMA dépendant du temps, Cahiers du C.E.R.0.,19,285-295.

Mélard, G.(1981), On an alternative model for intervention analysis, in O.D. Anderson and M.R. Perryman(eds)(1981),345-354.

Mélard, G.(1982a), On a deterministic sub-model for the innovation process in ARIMA models, 1981 Proceedings of the Business and Economic Statistics, American Statistical Association, Washington (to appear).

Mélard, G.(1982b), The likelihood function of a time-dependent ARMA model, in 0.D. Anderson and M.R. Perryman(eds) Applied time series analysis, North-Holland, Amsterdam, in press.

Mélard, G.(1982c), A fast algorithm for the exact likelihood of autoregressivemoving average models (submitted).

Mélard, G. and Kiehm, J.-L.(1980), Spécification d'un modèle ARIMA évolutif : approche par décomposition, Cahiers du C.E.R.0.,22,265-277.

Mélard, G. and Kiehm, J.-L. (1981), ARIMA models with time-dependent coefficients for economic time series, in 0.D. Anderson and M.R. Perryman(eds)(1981),355-363. Mélard, G. and Rouland, 0.(1979), Une méthode de sélection d'un algorithme de prévision à court terme, Communication aux Journées de Statistique de l'Association des Statisticiens Universitaires, Paris.

Newbold,P.(1981), Some recent developments in time series analysis, International Statistical Review, 49, 53-66. 